Proceedings

\title{
Room Temperature Ethanol Microsensors Based on Silanized Tungsten Oxide Nanowires ${ }^{\dagger}$
}

\author{
Stella Vallejos ${ }^{1,2 *}$, Zdenka Fohlerová ${ }^{1}$, Milena Tomić ${ }^{2}$, Isabel Gràcia ${ }^{2}$, Eduard Figueras ${ }^{2}$ and \\ Carles Cané 2 \\ 1 CEITEC-Central European Institute of Technology, Brno University of Technology, 61200 Brno, \\ Czech Republic; zdenka.fohlerova@ceitec.vutbr.cz \\ 2 Instituto de Microelectrónica de Barcelona (IMB-CNM, CSIC), Campus UAB, 08193 Bellaterra, Spain; \\ milena.tomic@imb-cnm.csic.es (M.T.); isabel.gracia@imb-cnm.csic.es (I.G.); \\ Eduard.Figueras@imb-cnm.csic.es (E.F.); carles.cane@imb-cnm.csic.es (C.C.) \\ * Correspondence: vargas@feec.vutbr.cz or stella.vallejos@imb-cnm.csic.es; Tel.: +420-5-4114-6153 \\ + Presented at the Eurosensors 2018 Conference, Graz, Austria, 9-12 September 2018.
}

Published: 22 November 2018

\begin{abstract}
Gas microsensors based on tungsten oxide ( $\left.\mathrm{WO}_{3-\mathrm{x}}\right)$ nanowires (NWs) silanized with APTES (3-aminopropyltriethoxysilane) are developed in this work. These surface modified microsensors are highly sensitive to ethanol at room temperature (RT) via photoactivation and show enhanced selectivity towards other volatile organic compounds (VOCs) including acetone and toluene.
\end{abstract}

Keywords: gas sensors; tungsten oxide; silanization

\section{Introduction}

Metal oxide (MOX) NWs are attractive in gas sensors because they have proved to enhance the functionality of these devices, particularly in terms of sensitivity, due to their higher surface area to volume ratio as opposed to bulk MOXs. These materials can be synthetized with tuned surface morphologies via scalable and industrially relevant bottom-up techniques. For instance, previously, we have demonstrated the synthesis of various $\mathrm{MOX}\left(\right.$ e.g., $\mathrm{WO}_{3-x}, \mathrm{ZnO}$, and $\mathrm{SnO}_{2}$ ) structures using a vapor-phase method enabled via aerosol-assisted (AA) chemical vapor deposition (CVD), and their enhanced sensitivity, stability and reproducibility to various gaseous analytes [1]. However, the lack of selectivity and need of relatively high temperatures, above $200{ }^{\circ} \mathrm{C}$, for proper operation of these materials were also evidenced, as for other MOX in the literature [2]. In this context, a next generation of gas sensor devices operating at low temperatures, ideally RT, and providing good selectivity to key target analytes, such as VOCs (ketones, hydrocarbons and aldehydes) is highly desirable to reduce the operational power consumption and achieve the demand of specific applications in terms of selectivity.

Photoactivation of gas sensitive materials can be used as an alternative to thermoactivation to circumvent high power consuming resistive heaters allowing gas sensing at RT [3]. Furthermore, the modification of the surface chemistry of inorganic semiconducting supports with organo functional molecules can also be used to enhance the selectivity and influence the electrical properties of the support. Previous studies in the field of modified surfaces for gas sensing have focused particularly on the surface modification of $\mathrm{Si}$ NWs and the determination of the characteristics of the molecular modifying layers to achieve better functionality [4]. However, the modification of gas sensitive MOX with organo functional molecules in the literature is still less common. Therefore, here we present our recent results on the fabrication of microsensors based on silanized $\mathrm{WO}_{3-\mathrm{x}} \mathrm{NWs}$. Silanization is a widely used method to modify MOX surfaces and encourage the formation of bonds across the 
interface between the MOX and organic compounds (e.g., VOCs). Hence, in this work we also study the sensing properties of the silanized microsensors towards VOCs such as ethanol, acetone and toluene using UV photoactivation at RT.

\section{Materials and Methods}

The microsensors consist of a membrane of $\mathrm{Si}_{3} \mathrm{~N}_{4} / \mathrm{SiO}_{2}(300 \mathrm{~nm} / 500 \mathrm{~nm}$ thick) with $\mathrm{Ti} / \mathrm{Pt}$ interdigitated electrodes ( $25 \mathrm{~nm} / 250 \mathrm{~nm}$ thick) on the top. $\mathrm{WO}_{3-\mathrm{x}} \mathrm{NWs}_{\mathrm{s}}$ were grown directly onto the interdigitated electrodes using the AACVD method described previously [3]. Subsequently, the gas sensitive NWs underwent a cleaning process, which included rinsing in deionized water, drying in desiccator, and finally removing the adsorbed species and generating active hydroxyl groups on the surface by plasma treatment. The silanization was carried out at $120^{\circ} \mathrm{C}$ via CVD. An aminosilane (APTES; 3-aminopropyltriethoxysilane, Sigma) solution was used to modify the sensitive films. The whole process spanned $10 \mathrm{~min}$. A photograph of the sensor device is displayed in Figure 1a.
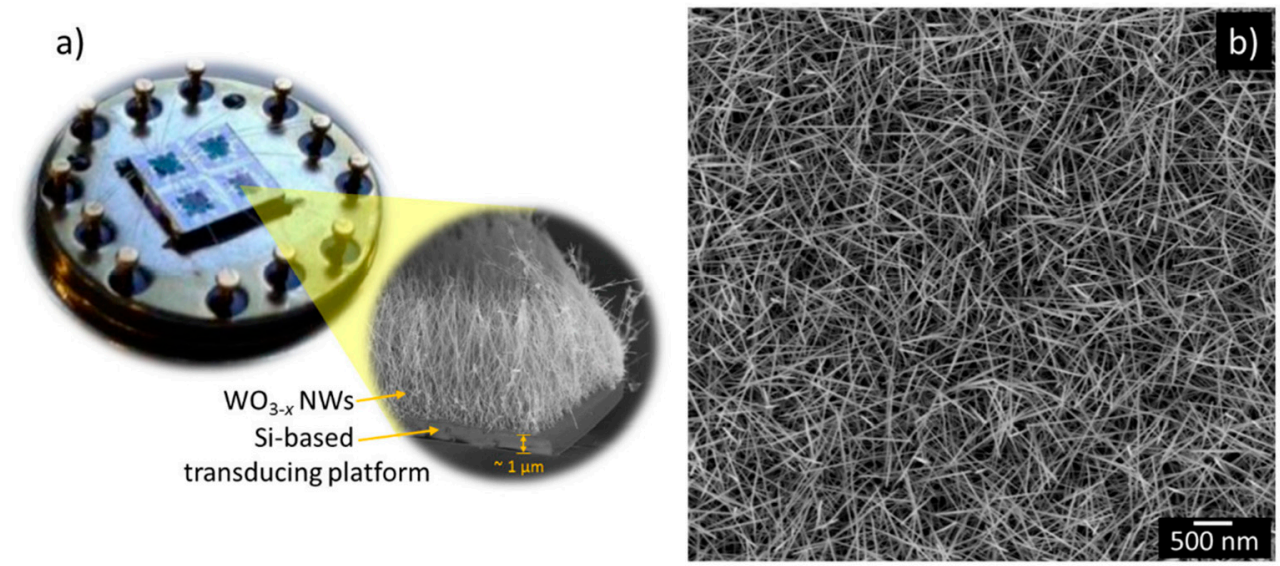

Figure 1. Photograph of the sensor device mounted on a TO-8 package and its cross-section after integration of the $\mathrm{WO}_{3-x} \mathrm{NWs}$ (a). SEM imaging (top-view) of the $\mathrm{WO}_{3-\mathrm{x}} \mathrm{NWs}(\mathbf{b})$.

The morphology of the films was examined using Scanning Electron Microscopy (SEM-Carl Zeiss, Auriga Series) and the chemical composition using X-ray photoelectron spectroscopy (AXIS SupraTM Kratos Analytical, with Al/Ag monochromatic X-ray source). Further analysis of the films were carried out using X-ray Diffraction (XRD-Rigaku SmartLab $3 \mathrm{~kW}, \mathrm{Cu} \mathrm{K} \alpha$ radiation) and transmission electron microscopy (HRTEM-FEI Tecnai F20, 200 kV).

Microsensors were tested in a continuous flow $(200 \mathrm{sccm})$ test chamber provided of mass flow controllers and continuous illumination from a LED (light-emitting diode) with wavelength of 365 $\mathrm{nm}$. Sensors were exposed to various calibrated concentrations of ethanol, acetone and toluene and subsequently the gaseous analytes were purged with air (3X, Praxair) until the initial baseline resistance in air was recovered. The sensor response was defined as $\mathrm{Ra} / \mathrm{Rg}$, where $\mathrm{Ra}$ and $\mathrm{Rg}$ are the resistance in air and in gas, respectively.

\section{Results}

SEM imaging of the films displayed NWs with diameters of $\sim 100 \mathrm{~nm}$ and lengths of $\sim 8 \mu \mathrm{m}$, corresponding to an aspect ratio of 80 (Figure 1). XRD analysis of the NWs showed crystalline structures with intense diffraction peaks at $23.1^{\circ} 2 \theta(\mathrm{d}=3.85 \AA)$ and $47.2^{\circ} 2 \theta(\mathrm{d}=1.92 \AA)$ that correspond the (002) and (004) reflections of the monoclinic phase $\mathrm{WO}_{3}(\mathrm{P} 21 / \mathrm{n}$; ICCD card no. 720677). Similarly, HRTEM also revealed highly ordered crystalline NW structures with marked planar spacing of $3.6 \AA$ along the NW, consistent with the unit cell observed by XRD and with previous results [2].

Figure 2 displays the XPS spectra for the APTES modified and non-modified $\mathrm{WO}_{3-\mathrm{x}} \mathrm{NWs}$. Both samples display $\mathrm{W} 4 \mathrm{f}$ core level peaks with similar characteristics to those observed in our previous 
works. Additionally, the spectrum recorded for the modified films shows the presence of Si 2p, Si $2 s$ and $\mathrm{N} 1$ 1s core-level peaks, thus demonstrating the silanization of the $\mathrm{WO}_{3-\mathrm{x}} \mathrm{NWs}$ surface.

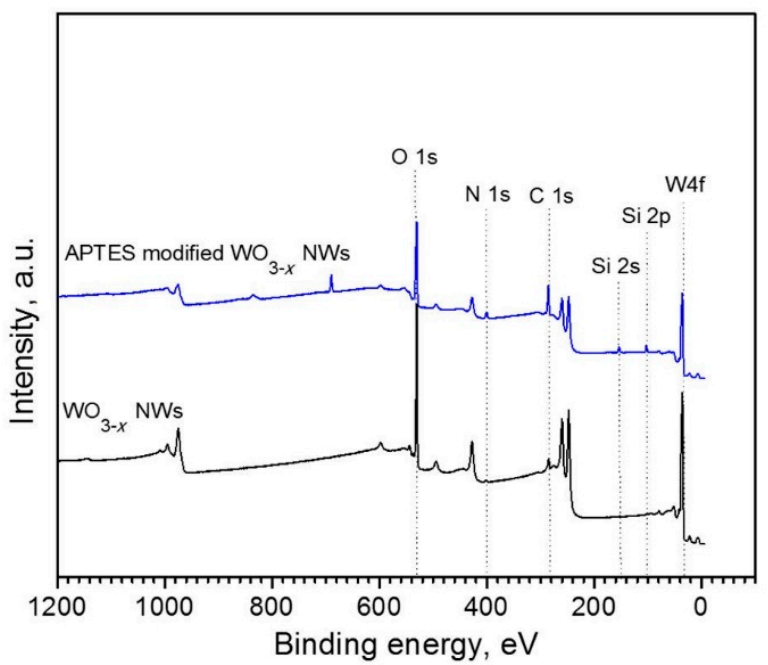

Figure 2. Survey XPS spectra for the APTES modified and non-modified $\mathrm{WO}_{3-\mathrm{x}} \mathrm{NWs}$ (only the peaks of interested have been marked).

Photoactivated gas sensing tests performed at RT towards various concentrations of acetone, ethanol and toluene demonstrated noticeable selectivity towards ethanol for the APTES modified $\mathrm{WO}_{3-\mathrm{x}} \mathrm{NWs}$ as opposed to the non-modified $\mathrm{WO}_{3-\mathrm{x}} \mathrm{NWs}$, which displayed negligible response to the gases tested (Figure 3a). The response of the APTES modified microsensors to ethanol reached ninefold the response of the non-modified microsensors for a concentration of $80 \mathrm{ppm}$ (Figure 3). The higher sensitivity of the APTES modified sensors to ethanol is attributed to the higher polarity of this molecule as compared to acetone and toluene [5].
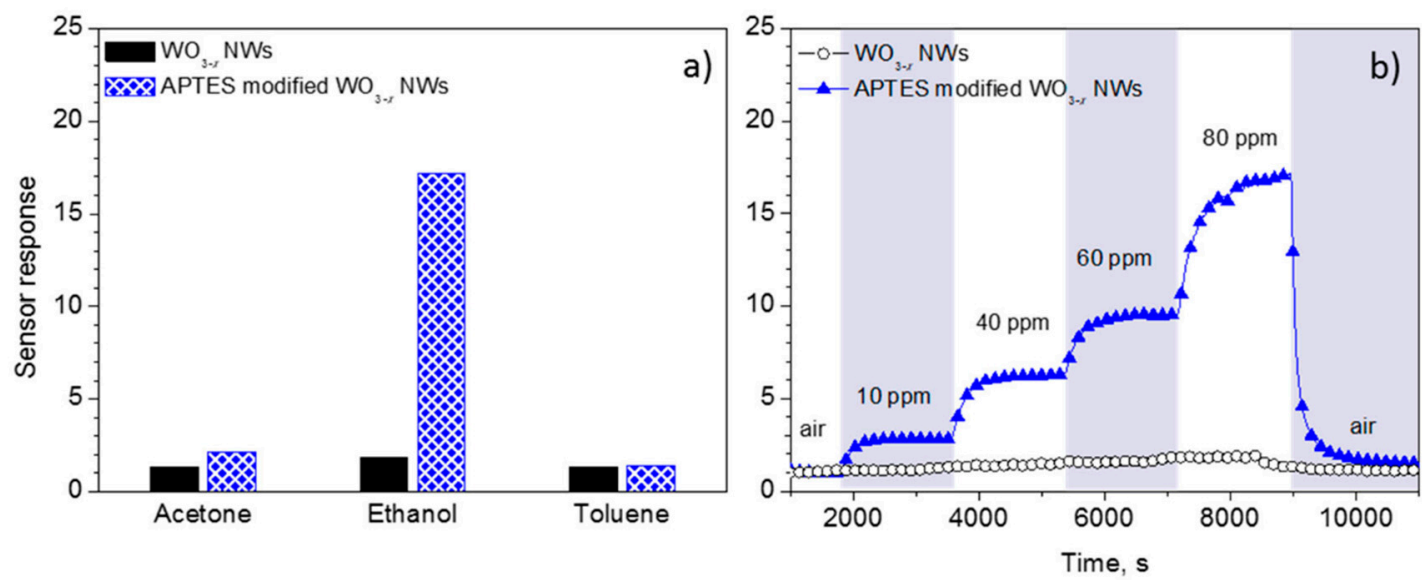

Figure 3. Sensor response to $80 \mathrm{ppm}$ of acetone, ethanol and toluene (a), and sensor response towards various concentrations of ethanol (b) for the APTES modified and non-modified $\mathrm{WO}_{3-\mathrm{x}} \mathrm{NWs}$.

\section{Conclusions}

Gas microsensors based on silanized $\mathrm{WO}_{3-x} \mathrm{NWs}$ have been developed in this work. Both, the synthesis of $\mathrm{WO}_{3-\mathrm{x}} \mathrm{NWs}$ and the silanization of their surface (using aminosilane) were performed directly on microtransducing platforms via CVD. XPS analysis of the modified $\mathrm{WO}_{3-\mathrm{x}} \mathrm{NWs}$ confirmed the silanization of their surface. Photoactivated gas sensing test at RT demonstrated that the silanized structures (as opossed to non-silanized structures) are highly sensitive and selective to ethanol towards other volatile organic compounds including acetone and toluene. 
Author Contributions: All authors conceived and designed the experiments; S.V., Z.F., M.T., and I.G. fabricated the sensor device and analyzed the gas sensitive material. S.V., I.G., E.F., and C.C. perform and analyzed the gas sensing test; S.V. wrote the paper.

Acknowledgments: This work has been supported by the Czech Science Foundation (GAČR) via Grant no. 1716531S. The support of the Spanish Ministry of Economy and Competitiveness via the Ramón y Cajal programme and projects TEC2015-74329-JIN-(AEI/FEDER, EU) and TEC2016-79898-C6-1-R (AEI/FEDER, EU) are also acknowledged. This research has made use of the infrastructures of the Spanish ICTS Network MICRONANOFABS partially supported by MINECO. CEITEC Nano Research Infrastructure (IDLM2015041, MEYS CR, 2016-2019) is also acknowledged.

Conflicts of Interest: The authors declare no conflict of interest. The founding sponsors had no role in the design of the study; in the collection, analyses, or interpretation of data; in the writing of the manuscript, and in the decision to publish the results.

\section{References}

1. Vallejos, S.; Umek, P.; Blackman, C. AACVD Control parameters for selective deposition of tungsten oxide nanostrucutres. J. Nanosci. Nanotechnol. 2011, 11, 8214-8220, doi:10.1166/jnn.2011.5027.

2. Vallejos, S.; Grácia, I.; Chmela, O.; Figueras, E.; Hubálek, J.; Cané, C. Chemoresistive micromachined gas sensors based on functionalized metal oxide nanowires: Performance and reliability. Sens. Actuator B 2016, 235, 525-534, doi:10.1016/j.snb.2016.05.102.

3. Prades, J.D.; Jimenez-Diaz, R.; Hernandez-Ramirez, F.; Barth, S.; Cirera, A.; Romano-Rodriguez, A.; Mathur, S.; Morante, J.R. Equivalence between thermal and room temperature UV light-modulated responses of gas sensors based on individual $\mathrm{SnO}_{2}$ nanowires. Sens. Actuator B 2009, 140, 337-341, doi:10.1016/j.snb.2009.04.070.

4. Wang, B.; Haick, H. Effect of Functional Groups on the Sensing Properties of Silicon Nanowires toward Volatile Compounds. ACS Appl. Mater. Interfaces 2013, 5, 2289-2299, doi:10.1021/am4004649.

5. Reichardt, C.; Welton, T. Solvents and Solvent Effects in Organic Chemistry: Third, Updated and Enlarged Edition; John Wiley \& Sons, Inc.: Hoboken, NJ, USA, 2003.

(C) 2018 by the authors. Licensee MDPI, Basel, Switzerland. This article is an open access article distributed under the terms and conditions of the Creative Commons Attribution (CC BY) license (http://creativecommons.org/licenses/by/4.0/). 